\title{
Liquid channel segregation and morphology and their relation with hot cracking susceptibility during columnar growth in binary alloys
}

\author{
Lei Wang ${ }^{\mathrm{a}, \mathrm{b}}$, Nan Wang ${ }^{\mathrm{a}, *}$, Nikolas Provatas ${ }^{\mathrm{b}, *}$ \\ ${ }^{a}$ Key Laboratory of Space Applied Physics and Chemistry, Ministry of Education, School of Science, Northwestern Polytechnical University, Xi'an, 710072, China \\ ${ }^{b}$ Department of Physics and Centre for the Physics of Materials, McGill University, Montreal, H3A 2T8,Canada
}

\begin{abstract}
The segregation and morphology of liquid channels at the last stage of solidification are closely related to hot cracking formation. We use Phase-Field (PF) simulations to investigate liquid channel characteristics during columnar growth of single-crystal and bi-crystal $\mathrm{Al}-\mathrm{Cu}$ alloys. It is found that the back-diffusion is weak in $\mathrm{Al}-\mathrm{Cu}$ alloys. Liquid channels in $\mathrm{Al}-2.0,3.0 \mathrm{wt} \% \mathrm{Cu}$ alloys coalesce into droplets above the eutectic temperature while the reduced Hot Cracking Susceptibility of Al-4.0wt\%Cu can be correlated to eutectic formation at the root of liquid channels. For bi-crystal growth, we demonstrate that non-equilibrium solute segregation at intergranular liquid channels (prospective Grain Boundaries (GBs)) depends on the convergent/divergent growth conditions between grains, and their misorientation angle. Divergent growth leads to stronger solute segregation as more solute diffuses laterally into divergent intergranular channels. It is thus expected that for the same GB energy, intergranular channels with higher solute composition can extend to lower temperatures, thus promote hot cracking. We introduce an approach for combining solid fraction vs. temperature curves calculated from microstructurally complex PF simulations with hot cracking models in the literature to predict the nominal composition with the highest HCS. Preliminary results from this work agrees well with experiments.
\end{abstract}

Keywords: Liquid channel, Segregation, Grain boundary, Phase-field, Hot cracking

\section{Introduction}

13

During columnar cellular/dendritic growth, the region where ${ }^{14}$ liquid and solid phases coexist due to segregation is termed as the mushy zone [1]. At the later stages of solidification, the microstructure forming inside the mushy zone is characterized by a dendritic solid skeleton with discontinuous liquid ${ }^{18}$ droplets and/or continuous channels distributed inside it. The ${ }^{19}$ liquid droplet and channels have quite different effects on the ${ }^{20}$ mechanical properties and hot cracking formation in the mushy ${ }^{21}$ zone. A solid network with liquid droplets behaves similarly to ${ }^{22}$ a coherent solid with slightly reduced strength and thus has a ${ }^{23}$ very low hot cracking susceptibility (HCS). A mushy zone with ${ }^{24}$

\footnotetext{
* Corresponding author.

Email addresses: leiwang1989@mail.nwpu.edu. cn (Lei Wang),

nan. wang@nwpu. edu. cn (Nan Wang),

provatas@physics.mcgill.ca (Nikolas Provatas)
}

Preprint submitted to Elsevier wide liquid channels can avoid hot cracking by adjusting its morphology by liquid feeding. However, when the last liquid that exists is in the form of very thin liquid channels, an opening in the mushy zone cannot generally be compensated by liquid feeding, and hot cracking occurs [2]. It can be concluded that the liquid channel morphology at the last stage of solidification, especially thin regions at the roots of liquid channels and grain boundaries (GBs) is critical to hot cracking.

An extensive body of experimental work has shown that the HCS of an alloy strongly depends on its nominal composition, which exhibits the typical $\Lambda$-shape [2, 3]. The composition-dependent solidification interval (equilibrium or non-equilibrium) provides a convenient index to quantify HCS 6 behaviour. Specifically, the larger the solidification range, the 27 longer the liquid channel and the higher tendency of cracking. 
cient, the solidification interval increases with the nominal com- 67 position initially and decreases after the formation of eutectics. 68 The maximum freezing range thus corresponds approximately 69 to the composition at which eutectics start to form. It is noted, 70 however, that while eutectic reactions can take place in small 71 liquid drops and liquid channels, it is the latter that are typically 72 considered to have a strong effect on hot cracking formation. $\quad 73$

Fig. 1 illustrates the trends on liquid channel morphology as a ${ }^{74}$ function of composition. For a dilute alloy containing only pri- 75 mary phase, solute segregation is weak and short liquid chan- 76 nels form (case a). Increasing the composition to a moderate 77 value leads to stronger segregation and longer liquid channels, ${ }^{78}$ with eutectic forming in isolated droplets (case b). Further in- 79 creasing the nominal composition increases solute segregation 80 further, leading to eutectic formation starting at the root of liq- 81 uid channels (case c). Eutectic forming between two colum- 82 nar dendrites (case c) is expected to prevent them from closing 83 in on each other, leaving wider liquid channels that can facil- 84 itate liquid feeding and thus reduce the HCS. The distribution 85 of metastable liquid below the eutectic temperature $\left(T_{e}\right)$ is thus ${ }^{86}$ important to study in order to better understand hot cracking. $\quad{ }_{87}$

Normally, the length of liquid channels between the 88 cells/dendrites can be characterized by the volume fraction of 89 solid phase, $f_{s}(T)$, which in mean field theories is typically cal- 90 culated by the Scheil equation. Here $f_{s}$ is the volume fraction 91 of solid phase and $T$ is temperature. However, the Scheil equa- 92 tion can only examine volume fraction and not where dendrites 93 coalesce. For this reason, coalescence is defined empirically to 94 occur at $f_{s}=0.94$ and the length of the liquid is usually deter- 95 mined to end at this point. The real image of where the colum- 96 nar dendrites join each other is still poorly understood, partic- 97 ularly if the coalescence point is higher or lower than the eu- 98 tectic temperature. Thus, a quantitative study that includes mi- ${ }_{99}$ crostructural details about dendritic coalescence is important to $_{100}$ show a real $\Lambda$ relationship for HCS. The importance of a precise ${ }_{101}$ $f_{s}(T)$ predictor lies in that it is an input into models for predi- ${ }_{102}$ cating HCS, such as RDG model (Rappaz-Drezet-Gremaud). ${ }_{103}$ In addition to the composition, another factor that can af-104 fect the length of liquid channel is grain boundary angle. As discussed by Rappaz [4] the grain boundary can vary from attractive to repulsive with the variation of misorientation angle. A repulsive grain boundary [4] is believed to be a necessary condition to induce hot cracking by extending the intergranular liquid channel to temperatures lower than the coalescence temperature inside a single grain, $T_{b}$. In addition to the GB energy, non-equilibrium solute enrichment can also affect liquid channels and emerging GB structures. Namely, higher solute content leads to liquid channels that extend to lower temperatures, for a fixed GB misorientation energy, and thus have a higher HCS. Luo et al. [5, 6] have shown that solute segregation can make a material brittle by forming intergranular liquid channels. The role of GB energy and GB solute enrichment is convolved in alloys. To identify the effect of solute segregation alone, it would be instructive to examine large misorientation angles for which GB energy is approximately constant. It is generally expected that divergent and convergent growth will give different levels of non-equilibrium segregation at intergranular channels and resultant GBs.

Non-equilibrium morphological and segregation details inside the mushy zone and at GBs are thus quite important in providing an accurate $f_{s}(T)$ for the analysis of hot cracking formation. The Phase-Field (PF) method, with the advantage of avoiding interface tracking, has been widely used to modelling microstructure development [4, 7- -10]. In addition, with the advent of accessible thin interface limit analyses coupled to parallel adaptive mesh refinement codes, the PF method has become a viable tool for handling large-scale simulations that can examine the development of the mushy zone in experimentally relevant systems for both single or polycrystalline columnar growth.

Using the Multi-Phase Field modelling approach, Böttger et al. have examined microstructure formation and its relationship with HCS during continuous casting of polycrystalline LowCarbon and High-Strength Low-Alloyed Steels [11-13]. Their simulations show that micro-alloying such as Ti and and related precipitates have a pronounced effect on HCS by increasing the 
coalescence temperature.

This work uses a recent quantitative Multi-Order Parameter ${ }_{143}$ phase field model of alloy solidification to study HCS in solidification under laser welding conditions in the $\mathrm{Al}-\mathrm{Cu}$ system, with the aim of isolating and characterizing the specific roles of solute on coalescence and eutectic formation in single crystal and bi-crystal columnar growth, as well as non-equilibrium segregation effects on the coalescence of bi-crystal grain boundaries. In the case of single crystal directional solidification, the effect of the nominal composition on liquid channel seg- ${ }^{144}$ regation and morphology is examined, and the results using the Scheil equation are compared to from PF simulations. In the case of bi-crystal directional solidification, the dependence of intergranular solute enrichment on convergent/divergent growth conditions and mis-orientation angle are characterized. Finally, we also couple analysis from our phase field simulations to the RDG and Kou models of hot cracking to specifically identify the nominal composition corresponding to the highest HCS in Al-Cu alloys. Our results demonstrate that the effect of the nominal composition, divergent/convergent growth conditions and misorientation angle between bi-crystals, can have a significant effect on solute segregation and, thus, hot cracking formation in welding processes.

\section{Phase Field Model of Polycrystalline Solidification}

To investigate solute segregation at liquid channels and emerging GBs, the quantitative multi-order parameter PF model ${ }_{145}$ of polycrystalline binary alloys developed by Ofori-Opoku et ${ }_{146}$ al. [14] was used. In the model, $c$ represents solute concentra- ${ }_{147}$ tion and $T$ is the temperature. Each grain, $i$, is represented by ${ }_{148}$ an order parameter $\phi_{i}$, which tracks the solid-liquid interface ${ }_{149}$ of a given grain. Nominally, each $\phi_{i}=1$ in the bulk of the $i^{t h}{ }_{150}$ grain, while $\phi_{i}=0$ in the liquid or in the bulk of any other grain ${ }_{151}$ $j \neq i$. The interactions between order parameters are defined ${ }_{152}$ such that $\phi_{1}+\phi_{2}+\cdots \phi_{N}<1$ everywhere. (Practically, we work with interpolation functions that allow $\phi_{i}$ to vary between $\left.-1<\phi_{i}<1\right)$. Ordering in the system is completely defined everywhere by the set $\left\{\phi_{i}\right\}$, which is compactly represented by a phase field vector $\vec{\phi}=\left(\phi_{1}, \phi_{2}, \cdots, \phi_{N}\right)$, where $N$ is the total number of grains in the system.

\subsubsection{Free energy functional}

In terms of $c,\left\{\phi_{i}\right\}$ and $T$, the free energy functional of a solidifying alloy is

$F(\vec{\phi}, c, T)=\int d \mathbf{x}^{3}\left\{\frac{1}{2} \sum_{i}\left|\epsilon(\vec{\phi}) \vec{\nabla} \phi_{i}\right|^{2}+f(\vec{\phi}, c, T)+f_{i n t}(\vec{\phi})\right\}$,

where $\mathbf{x}$ is a spatial variable. Here, $f_{\text {int }}(\vec{\phi})$ controls the interaction between the $\phi_{i}$ and prevents overlap in the bulk of grains. It form is given by

$$
f_{i n t}(\vec{\phi})=\sum_{i} \sum_{j \neq i} \alpha_{i j}\left(\frac{1+\phi_{i}}{2}\right)^{2}\left(\frac{1+\phi_{j}}{2}\right)^{2}
$$

where $\alpha_{i j}$ is an energy density, set here to a constant $\left(\alpha_{i j}=\alpha\right)$. The remainder of the bulk free energy follows the form

$$
f(\vec{\phi}, c, T)=H f_{D}(\vec{\phi})+f_{i d}(\vec{\phi}, c, T)
$$

where $f_{D}(\vec{\phi})=\sum_{i}\left(-\phi_{i}^{2} / 2+\phi_{i}^{2} / 4\right)$ sets a barrier between each $\phi_{i}$ and the liquid, and $H$ defines a nucleation barrier energy scale $\left([H]=\mathrm{J} / \mathrm{m}^{3}\right)$. The function $f_{i d}(\vec{\phi}, c, T)$ is the standard free energy of an ideal binary alloy, written explicitly in polycrystalline form in Ref. [14]. The function $\epsilon(\vec{\phi})$ in Eq. [1) is a gradient energy coefficient $\left.\left(\left[\epsilon^{2}\right]=\mathrm{J} / \mathrm{m}\right]\right)$ that is modulated by the value of the order parameter set $\left\{\phi_{i}\right\}$ to take on different values for solid-liquid and solid-solid interfaces.

The dynamics of the order parameter $\phi_{i}$ are defied through the minimization of the functional in Eq. 11, namely $\partial \phi_{i} / \partial t=$ $-M \delta F / \delta \phi_{i}$, where $M$ is a mobility governing the order pa- 
rameter kinetics. In expanded form this equation becomes

$$
\begin{aligned}
\tau(T) \frac{\partial \phi_{i}}{\partial t}= & \vec{\nabla} \cdot\left(W(\vec{\phi})^{2} \vec{\nabla} \phi_{i}\right) \\
& +\partial_{x}\left(\left|\vec{\nabla} \phi_{i}\right|^{2} W(\vec{\phi}) \frac{\partial W(\vec{\phi}}{\partial\left(\partial_{x} \phi_{i}\right)}\right) \\
& +\partial_{y}\left(\left|\vec{\nabla} \phi_{i}\right|^{2} W(\vec{\phi}) \frac{\partial W(\vec{\phi}}{\partial\left(\partial_{y} \phi_{i}\right)}\right) \\
& +\phi_{i}-\phi_{i}^{3} \\
& -\bar{\lambda}\left(1-\phi_{i}^{2}\right)^{2}\left(U+\frac{T-T_{0}}{|m|(1-k) c_{l}^{0}}\right) \\
& -\omega \frac{1+\phi_{i}}{2} \sum_{j \neq i}\left(\frac{1+\phi_{j}}{2}\right)^{2},
\end{aligned}
$$

where $W(\vec{\phi})=\epsilon(\vec{\phi}) / \sqrt{H}=W_{0} a(\vec{\phi})$, with $W_{0}$ the characteristic length of the solid-liquid interface and $a(\vec{\phi})$ a dimensionless gradient energy function, while $\bar{\lambda}=R T(1-k)^{2} c_{l}^{0} /\left(\Omega_{o} H\right){ }^{156}$ $\omega=\alpha / H$, and $\left(T_{0}, c_{l}^{0}, m, k, \Omega_{o}\right)$ are, respectively, a refer- ${ }^{157}$ ence temperature, the solute composition on the liquid side of $a^{158}$ steady-state planer interface at $T_{0}$, the liquidus slope, the solute ${ }^{159}$ partition coefficient of the alloy, and the molar volume of the material. Also, $t$ is time and $\tau(T)=\tau_{o} \bar{\tau}(T)$, where, following Ref. [15],

$$
\bar{\tau}(T)=\left[1-\frac{T-T_{0}}{|m| c_{l}^{0}}\right] a^{2}(\vec{\phi}),
$$

and $\tau_{0}$ sets a kinetic time scale defining fluctuations in $\phi_{i}$. The supersaturation field $U$ defined by

$$
U=\frac{1}{1-k}\left\{\frac{2 c / c_{l}^{0}}{1+k-(1-k) \sum_{i} \phi_{i}}-1\right\}
$$

Here, the constant $\bar{\lambda}$ couples $\phi_{i}$ to $U$, and is considered a con- ${ }_{164}$ vergence parameter to achieve correspondence with sharp inter- ${ }_{165}$ face kinetics.

The dynamics of the supersaturation $U$ are governed by

$$
\begin{aligned}
\left(\frac{1+k}{2}-\frac{1-k}{2} \phi\right) \frac{\partial U}{\partial t} & =\vec{\nabla} \cdot\left(D_{l} q(\vec{\phi}) \vec{\nabla} U-\vec{j}_{a t}\right) \\
& +[1+(1-k) U] \frac{1}{2} \sum_{i} \frac{\partial \phi_{i}}{\partial t}
\end{aligned}
$$

where $q(\vec{\phi})$ modulates solute diffusion across interfaces. Its form is given by

$$
q\left(\vec{\phi}_{i}\right)=\frac{1-\psi}{2}+\frac{(1+\psi)[1+k+(1-k) \psi]}{4} \frac{D_{s}}{D_{l}}
$$

where $\psi=\sum_{i}^{N} \phi_{i}+(N-1)$, and $D_{l}$, and $D_{s}$ liquid diffusion coefficient, and solid diffusion coefficient, respectively. The term $\vec{j}_{a t}$ is a non-variational term called the anti-trapping flux. It is added in as part of a strategy for the any solid-liquid interface defined by $\phi_{i}$ to emulate the sharp-interface model for alloy solidification in the limit of diffuse interfaces. Its form was generalized from Ref. [15] to

$$
\vec{j}_{a t}=-\frac{1}{2 \sqrt{2}}[1+(1-k) U] \sum_{i} \frac{\partial \phi_{i}}{\partial t} \frac{\vec{\nabla} \phi_{i}}{\vec{\nabla} \phi_{i}}
$$

The term $\vec{j}_{a t}$ becomes negligible for solid-solid interfaces and at vanishing solidification rates. It is noted that the concentration $c_{l}^{0}$ is related to the nominal composition of the binary alloy, $c_{0}$, by $c_{l}^{0}=c_{0} / k$.

Temperature is modelled in the phase field simulations by a frozen temperature approximation corresponding to Bridgman directional solidification. Its form is given by

$$
T(y, t)=T_{0}+G\left(y-V_{p} t\right)
$$

where $G$ is the thermal gradient and $V_{p}$ is the pulling velocity.

All calculations with the above model, and results shown, assume that space and time are dimensionless through the rescaling $x \rightarrow x / W_{0}$ and $t \rightarrow t / \tau_{0}$, respectively. The relationship between the parameters $W_{o}, \tau_{0}$ and $\bar{\lambda}$ is discussed further below. Our simulations were carried out in 2 dimensions.

\subsection{Modeling solid-liquid interfaces}

The PF model parameters $\left(W_{0}, \tau_{0}\right)$ are selected such that the kinetics of any free solid-liquid interface defined by any $\phi_{i}$ reproduce the boundary conditions of the sharp-interface solidification model, in the limit of diffuse interfaces [15]. Namely, $\left(W_{o}, \tau_{o}\right)$ are chosen such that the isotropic components of the chemical capillary length, $d_{0}$, and the interface kinetic coeffi- 
cient, $\beta$, are related to the PF parameters by

$$
\begin{gathered}
d_{0}=\frac{\Gamma}{\left(|m|(1-k) c_{l}^{0}\right)}=\frac{a_{1} W_{0}}{\bar{\lambda}} \\
\beta=\frac{a_{1} \tau_{0}}{\bar{\lambda} W_{o}}\left[1-a_{2} \frac{\bar{\lambda} W_{0}^{2}}{\tau_{0} D_{l}}\right],
\end{gathered}
$$

\subsection{Modeling grain boundaries}

along any solid-liquid interface defined by any $\phi_{i}$, where $a_{s}={ }_{188}$ $1-3 \epsilon_{4}$ and $\psi$ is the angle of the interface normal of each ${ }_{189}$ grain with the x-axis. The small parameter $\epsilon_{4}$ is the anisotropy ${ }_{190}$ strength. To interpolate the function $a(\vec{\phi})$ continuously between ${ }_{191}$ its form in Eq. (13) and its form at solid-solid interfaces (see below), the parameter $\mathcal{E}=\left(\phi_{i}+1\right)^{2} \sum_{j \neq i}\left(\phi_{j}+1\right)^{2}$ is used. $\operatorname{In}_{193}$ particular, if $\mathcal{E}<\epsilon^{\prime}\left(\epsilon^{\prime}\right.$ is a small number) at any point of any $\phi_{i}{ }_{194}$ Eq. 13 is applied, otherwise $\mathcal{E} \geq \epsilon^{\prime}$ corresponds to solid-solid ${ }_{195}$ interface between $\phi_{i}$ and one or more $\phi_{j}, j \neq i$.

When updating the equation of motion for any $\phi_{i}$, if the cri-200 terion $\mathcal{E} \geq \epsilon^{\prime}$ (see previous section) is satisfied for any posi-201 tion and any $j \in\{1,2, \cdots, N\}$, this is taken to correspond to202 a solid-solid interface between grain $i$ and grain $j$. When $\vec{\phi}_{i^{203}}$ satisfies the above condition along any $i-j$ interface, the dimen-204 sionless gradient energy coefficient becomes

$$
a(\vec{\phi}) \rightarrow M\left[\vartheta_{i j} / \theta_{m}\right]\left[1-\ln \left(\vartheta_{i j} / \theta_{m}\right)\right] \mathcal{A}(\psi)
$$

where $\vartheta_{i j}$ denotes the misorientation angle between grain $i$ and grain $j$, and $M$ is a dimensionless scaling factor, set to $M=1$ in this work. $\theta_{m}$ is the angle at which GB energy reaches a maximum. Equation (14) describes the Read-Shockley grain boundary energy form [17]. For practicality, the function

$$
\theta(\vec{\phi})=\frac{\sum_{i j} \vartheta_{i j}\left(\phi_{i}+1\right)^{2}\left(\phi_{j}+1\right)^{2}}{\sum_{i j}\left(\phi_{i}+1\right)^{2}\left(\phi_{j}+1\right)^{2}}
$$

is used to calculate mis-orientation angle locally in $a(\vec{\phi})$.

For a grain boundary defined by two order parameters, $\phi_{i}$ and $\phi_{j}$, the interface energy, $\gamma_{g b}$, is determined by

$$
\begin{array}{r}
\gamma_{g b}(\theta)=H W_{0} \int_{-\infty}^{\infty}\left[\frac{1}{2} \sum_{i} a^{2}(\vec{\phi})\left(\frac{d \phi_{0}^{i}}{d \xi}\right)^{2}+\sum_{i=1}^{2} f_{D}\left(\phi_{0}^{i}\right)\right. \\
\left.+2 \omega\left(\frac{\phi_{0}^{i}+1}{2}\right)^{2}\left(\frac{\phi_{0}^{j}+1}{2}\right)^{2}-f_{D}^{\text {bulk }}\right] d \xi
\end{array}
$$

where $a(\vec{\phi})$ is given by Eq. $14, \xi=x / W_{0}$ and $\left(\phi_{i}^{o}, \phi_{j}^{o}\right)$ correspond to the steady state solutions of the phase field equations of an $i-j$ bi-crystal in $1 \mathrm{D}$, which can be computed numerically [14]. Since the results presented here are not sensitive to the precise value of $\gamma_{g b}$, the functions $\phi_{i}^{o}$ and $\phi_{j}^{o}$ are taken, for simplicity, as the usual hyperbolic tangent profile solutions. When $\theta=\theta_{m}, a(\vec{\phi})=\mathcal{A}(\psi)$, yielding the maximum grain boundary energy, denoted $\gamma_{g b}\left(\theta_{m}\right)=\gamma_{g b}^{\max }$. The solid-liquid interface energy is given by $\gamma_{s l}=a_{1} H W_{0}$. Thus, Eq. (16) scales the GB energy by the corresponding solid-liquid surface energy defined by $W_{o} H$.

The interaction $\omega$ can set under the assumption of negligible equilibrium adsorption at a steady-state solid-solid interface. Following Ref. [14], this is achieved by setting $\omega=$ $A \bar{\lambda}\left(T-T_{s}\right) /\left(T_{s}-T_{l}\right)$, where $A$ is a proportionality constant, determined numerically here to be $A \approx 8$. Note that the GB adsorption mentioned here is the composition difference of GBs 
from bulk composition at equilibrium. Kinetic processes such ${ }_{234}$ as solidification and grain growth can also cause composition ${ }_{235}$ differences which is referred to as non-equilibrium segrega-236 tion/enrichment [18].

The considerations above reveal that the ratio $\gamma_{g b}(\theta) / \gamma_{s l}$ de-238 pends on the driving force coefficient $\Delta \equiv \bar{\lambda}\left(T-T_{s}\right) /\left(T_{s}-T_{l}\right){ }^{239}$ The dependence of $\gamma_{g b}^{\max }$ on $\Delta$ and $\gamma(\theta)$ vs. $\theta$, for a particu-240 $\operatorname{lar} \Delta$, were calculated numerically and are shown in Fig. 2 (a) ${ }^{241}$ and (b), respectively. In the simulations reported here, we set ${ }^{242}$ $\bar{\lambda}=0.2$ along any solid-solid interface, and calculated $\gamma^{\max _{243}}$ based on the temperature $T=750 \mathrm{~K}$. For this choice of $\bar{\lambda},{ }^{244}$ this corresponds to a grain boundary energy many times larger245 than the solid-liquid energy, which was assumed for simplic-246 ity. Different grain boundary energies can be attained by tuning ${ }^{247}$ the $A, \bar{\lambda}$ and $T$. It is noted also, that attractive and repulsive ${ }^{248}$ grain boundaries can be modelled by varying the misorienta-249 tion (Fig. 2(b)).

\subsection{Implementing noise in simulations}

Compositional fluctuations induced by thermal noise are in- ${ }^{254}$ cluded in simulations by adding a stochastic mass flux to $U^{255}$ evolution equation (Eq. (7) ), the statistics of which satisfy

$$
<J_{u}^{m}(\vec{r}, t) J_{u}^{m}\left(\overrightarrow{r^{\prime}}, t\right)>=2 D q(\phi) F_{u} \delta\left(\vec{r}-\overrightarrow{r^{\prime}}\right) \delta\left(t-t^{\prime}\right)
$$

Following Ref. [19], the magnitude of the fluctuations is given 260 by $F_{u}=F_{u}^{0}[1+(1-k) U]$, where $F_{u}^{0}=k \Omega_{0} /(1-k)^{2} N_{A} c_{0}, 261$ $N_{A}$ is Avogadro's number and $\Omega_{0}$ is molar volume of solvent262 atoms. In numerical simulations, noise amplitude is scaled with ${ }_{263}$ the grid spacing. Details of the noise implementation can be 264 found in [19]. It is found that the simulation microstructures265 are mainly cellular even with noise included, which is exper-266 imentally consistent [20]. This can be attributed to small pri-267 mary spacing due to high growth velocity and thermal gradient.268 For divergent growth, the space between two grains also adopts269 side-branches.

\subsection{Material, solidification conditions}

The well-characterized Al-Cu alloy system was selected in this work with the physical properties listed in Table 1 . The liquidus and solidus are nearly straight lines with $m$ and $k$ nearly constant. Nominal compositions of 2.0, 3.0, and $4.0 \mathrm{wt} \% \mathrm{Cu}$ were studied. We used a thermal gradient $G=1 \times 10^{6} \mathrm{~K} / \mathrm{m}$ and an isotherm velocity $V_{p}=5 \mathrm{~mm} / \mathrm{s}$, which are typical solidification conditions in lower speed laser/electron beam welding processes. For metals and non-faceted alloys, interface non-equilibrium effects typically become important when the growth velocity is greater than $10 \mathrm{~mm} / \mathrm{s}$. Therefore, the atomic attachment effect and solute trapping effect are neglected here. For all calculations, the minimum grid spacing $d x / W_{0}=0.8$ was used and the total system size is $180 \mu \mathrm{m}$ in growth direction and $108 \mu \mathrm{m}$ in width direction. The phase-field equations are solved with a finite difference method implemented on an adaptive mesh refinement algorithm analogous to the original version by Provatas et al. [21, 22].

\section{Results}

Fig. 3 shows liquid channel morphologies during columnar growth of $\mathrm{Al}-2.0,3.0$, and $4.0 \mathrm{wt} \% \mathrm{Cu}$ alloys under the same solidification conditions, with four isotherms at temperatures $T_{m}$ (melting), $T_{l}$ (liquidus), $T_{s}$ (solidus), and $T_{e}$ (eutectic) indicated. The microstructures appear to be cellular in most cases. As with the primary spacing, there is a variability in liquid channel length. When only the primary reaction is considered, the length of liquid channels increases with increasing nominal composition from $2.0 \%$ to $4.0 \mathrm{wt} \% \mathrm{Cu}$. However the liquid below $T_{e}$ becomes metastable and will transform to eutectic. In this work, we assume this metastable liquid $\rightarrow$ eutectic reaction can occur fairly close to $T_{e}$ and in a short time scale. Therefore, the metastable liquid can be approximately regarded as the reverse of non-equilibrium eutectic phase.

The solid fraction vs. temperature, $f_{s}(T)$ curves calculated from PF simulations and from the Scheil equation for Al-2.0, 3.0, and 4.0wt\% Cu alloys are shown in Fig. 4 . Both PF simu- 
nominal composition and agree well with each other at low 308 $f_{s}$ range. At high $f_{s}, \mathrm{PF}$ simulations give higher $f_{s}(T)$ for азоя given temperature, predicting the end of primary solidification ${ }_{310}$ at higher temperatures (e.g. Al-2.0wt\% Cu), and a lower frac-311 tion of non-equilibrium eutectics (e.g. Al-3.0, 4.0wt\%Cu). 312

Fig. 5 shows the $f_{s}(T)$ curves for Al-3.0wt\% Cu alloys from ${ }_{313}$ PF simulations with different solid diffusion coefficients. The 314 solid diffusion is seen to have a strong effect on $f_{s}(T)$ at high $f_{s^{315}}$ range and at low temperature. The difference between $D_{s}=0_{316}$ and $3.0 \times 10^{-13} \mathrm{~m}^{2} / \mathrm{s}$ is minimal. With the increase of solid ${ }_{317}$ diffusion coefficient from with $3.0 \times 10^{-13}$ to $3.0 \times 10^{-11} \mathrm{~m}^{2} / \mathrm{s}, 318$ back-diffusion affects the $f_{s}(T)$ curve substantially.

Fig. 6 shows the liquid channel morphologies in colum-320 nar growth of a diverging bi-crystal in $\mathrm{Al}-2.0 \mathrm{wt} \% \mathrm{Cu}$ and $\mathrm{Al}-321$ $4.0 \mathrm{wt} \% \mathrm{Cu}$ alloys. The divergent bi-crystals have a misorienta-322 tion angle of $20^{\circ}$. Regions just below $T_{e}$ are enlarged to shows2з structural details of the liquid pools. The intergranular liquid 324 channel in the Al-2.0wt\% Cu alloy merges above $T_{e}$ and forms325 a continuous $\mathrm{GB}$. In the $\mathrm{Al}-4.0 \mathrm{wt} \% \mathrm{Cu}$ alloy, metastable liquid ${ }_{326}$ exists below $T_{e}$, and only part of intergranular channel starts to327 overlap.

The solute distribution maps for convergent and divergent 329 growth of $\mathrm{Al}-2.0 \mathrm{wt} \% \mathrm{Cu}$ bi-crystals is presented in Fig. 7 For the same misorientation, the divergent growth leads to higher solute composition in the intergranular liquid channels compared to convergent growth. The dependence of this nonequilibrium solute segregation at GBs versus misorientation is plotted in Fig. 8 for the data of Fig. 7 . We defined grain boundaries as areas in the data where the two order-parameter fields start to overlap, based on the criterion discussed in the model section. Segregation is seen to increase with misorienation for converging growth conditions, while it decreases with misorientation for divergent growth conditions.

\section{Discussion}

\subsection{Liquid channels in single crystal columnar growth}

The segregation in liquid channels can be influenced strongly $y_{34}$ by the alloy nominal composition. For a given alloy with $\mathrm{a}_{345}$ constant solute partition coefficient, $k$, solute segregation increases with the nominal composition. Increasing the nominal composition gives rise to inter-dendritic/cellular liquid channels which contain more solute and can remain stable at lower temperatures. This can be seen in Fig. 3. One way to describe segregation in liquid channels is through $f_{s}(T)$, which can be calculated from either microsegregation models or PF simulations. When the temperature is below $T_{e}$, any remaining liquid becomes metastable and will transform to eutectic phase. Assuming eutectic reaction happens close to $T_{e}$ and back-diffusion is weak, the fraction of eutectic can be approximated by $f_{e}=1-f_{s}\left(T_{e}\right) . f_{s}(T)$ curves can also be calculated from the Scheil equation. Several approximations are made in this formalism, namely, zero back-diffusion, infinitely rapid liquid diffusion, and planer solid-liquid interface. In terms of the nominal composition effect on segregation, the Scheil equation predicts similar results as $\mathrm{PF}$ simulations at small $f_{s}$. However, the Scheil equation predicts higher liquid fraction $\left(f_{l}\right)$ in the high $f_{s}$ range (Fig. 4). A possible reason for this discrepancy can be due to the fact back-diffusion is neglected in the Scheil equation, which modifies $f_{s}(T)$ by reducing solute segregation in the liquid.

To further examine the role of back-diffusion effect on $f_{s}(T)$, PF simulations with different solid-diffusion coefficient, $D_{s}$ are preformed and the result is shown in Fig. 5. Increasing the solid state diffusion coefficient increases the back-diffusion substantially, with the difference between the curves becoming larger with undercooling below $T_{s}$. For Al-Cu alloys considered here, $D_{s} \approx 3.0 \times 10^{-13} \mathrm{~m}^{2} / \mathrm{s}$ and back-diffusion has a minimal effect on $f_{s}(T)$, as in the Scheil model with $D_{s}=0$. However, other $\mathrm{Al}$ alloys such as $\mathrm{Al}-\mathrm{Mg}$ and $\mathrm{Al}-\mathrm{Zn}$ have a much lower $T_{e}$, above which the liquid can remain stable. In those cases, the driving force for back-diffusion can be large and affect $f_{s}(T)$ curves substantially assuming constant $D_{s}[4,14]$. Therefore, back-diffusion needs to be considered when studying the HCS of alloys with low $T_{e}[23,24]$. In this work, the main reason for the difference in $f_{s}(T)$ between the Scheil equation and PF simulations can be attributed to the variability of liquid channel 
lengths and interface curvatures, which makes the PF composition at interfaces inside channels deviate from the equilibrium composition of phase diagram and thus affect $f_{s}(T)$.

In addition to $f_{s}(T)$, it is also important to consider the spatial morphology and distribution of liquid. One feature of importance in this case would be the value of the eutectic temperature, $T_{e}$ relative to the coalescence temperature, $T_{b}$. Liquid below $T_{e}$ is metastable and transforms to eutectic phase. This can happen over a short time scale and can change the liquid morphology substantially. Otherwise the liquid channels can coalescence into isolated droplets at $T_{b}$. Therefore the metastable liquid can be either inside liquid channels or in the form of ${ }^{378}$ droplets, depending on the relative values of $T_{b}$ and $T_{e}[25]{ }^{379}$ When $T_{b}>T_{e}(\mathrm{Al}-3.0 \mathrm{wt} \% \mathrm{Cu})$, thin liquid channels typically ${ }^{380}$ transform to liquid droplets first and the eutectic reaction takes ${ }^{381}$ place inside these droplets. For Al-4.0wt $\% \mathrm{Cu}, T_{b}<T_{e}$, which ${ }^{382}$ implies that metastable liquid $\rightarrow$ eutectic reaction will predom- ${ }^{383}$ inately take place inside liquid channels. In this case, eutec- ${ }^{384}$ tics can form continuously and a so-called "continuous eu- ${ }^{385}$ tectic" can be observed. This is shown by Liu et al. very ${ }^{386}$ recently [26] in welds of $2014 \mathrm{Al}$ (an alloy with composi- ${ }^{387}$ tions close to $4.0 \mathrm{wt} \% \mathrm{Cu}$ ). This is consistent with Fig. 3(c), in ${ }^{388}$ which wider liquid channels in $\mathrm{Al}-4.0 \mathrm{wt} \% \mathrm{Cu}$ will form eutec- ${ }^{389}$ tic phases between the two columnar dendrites and prevent the ${ }^{390}$ formation of thin liquid channels, thus permitting liquid feed- ${ }^{391}$ ing, which decreases HCS. Reduced HCS of Al-4.0\% Cu alloys has been observed experimentally. Katgerman et al. [27, 28] ${ }^{393}$ studied the effect of composition on hot cracking during chill ${ }^{394}$ casting of Al-Cu alloys, also observing no hot cracking for al- ${ }^{395}$ loys with $\mathrm{Cu}$ content higher than 4.0.

\subsection{Intergranular liquid channels in bi-crystal growth}

Hot cracking is normally observed at GBs. The GB effect on hot cracking can be explained by repulsive GBs extending intergranular liquid channels to much lower temperatures than ${ }_{401}$ $T_{b}$. The energy of tilted GB, $\gamma_{g b}$ increases with misorientaion,402 reaching the maximum value at $\theta=\theta_{m}$ and remaining constant ${ }_{403}$ (Fig. 2(b)). During the merger of intergranular liquid channels,404 two solid-liquid interfaces are replaced by one GB. For smallangle GBs, $\gamma_{g b}<2 \gamma_{s l}$, and there is a free energy decrease in formation of a GB, favouring intergranular liquid channel merger at $T=T_{b}$, the same temperature as for liquid channels in a single grain. For large misorientation, $\gamma_{g b}>2 \gamma_{s l}$, and a larger undercooling is required to provide a driving force for GB merger. This undercooling is given by

$$
\Delta T_{b}(\theta)=\frac{\gamma_{g b}(\theta)-2 \gamma_{s l}}{\Delta S_{f} \delta}
$$

where $\Delta S_{f}$ is the volumetric entropy of fusion and $\delta$ is the width of solid-liquid interface. $\delta$ is not well-defined but is on the order of nanometers. In this work, we only consider high-angle GBs and $\Delta T_{b}=\Delta T_{b}\left(\theta_{m}\right)$ will be used hereafter. The specific value of $\Delta T_{b}$ is material-dependent. However, this value is believed to be large for alloys. $\Delta T_{b}=83 \mathrm{~K}$ is predicted for MC2 superalloys, as shown by Wang et al. [29]. More recently, by using High-resolution transmission electron microscopy and Auger spectroscopy, Luo et al. [5] observed nano-scale liquid layers at $95 \mathrm{~K}$ below the bulk eutectic temperature. $\Delta T_{b}=48 \mathrm{~K}$ can be estimated for Al-Cu alloys when $\gamma_{g b}=2.2 \gamma_{s l}$ and $\delta=1 \mathrm{~nm}$ are used. This undercooling is large enough to extend the intergranular liquid channels in Al3.0,4.0wt $\% \mathrm{Cu}$ alloys below $T_{e}$, i.e. $T_{b}-\Delta T_{b}<T_{e}$. A large $\Delta T_{b}$ implies that high-angle GBs can remain wet at the temperature between $T_{b}$ and $T_{b}-\Delta T_{b}$, a temperature range where most liquid regions have solidified. This thin GB liquid layer can reduce the strength and is hard for liquid feeding, which makes $T_{b}-\Delta T_{b}<T<T_{b}$ the most vulnerable range to form hot cracking. It is instructive here to distinguish full liquid structure (blue color in Fig. 6) from the nano-scale liquid layers formed at wet GBs, which are considered to be partially-ordered (yellow color) "liquid-like" structure [5]. We will refer this "liquid like" structure as GB liquid layer.

While the lowest temperature at which GBs can remain wet is $T_{b}-\Delta T_{b}$, whether or not the intergranular liquid channel can extend to this lowest temperature depends on the structure formed between two grains. This point can be illustrated 
clearly in Fig. 6. In the Al-2.0wt\%Cu alloys (Fig. 6(a)), where ${ }_{443}$ intergranular channels start to merge to form wet GB for tem-444 peratures above $T_{e}$, which can stay wet until $T_{b}-\Delta T_{b}$. $\operatorname{In}_{445}$ contrast, in the $\mathrm{Al}-4.0 \mathrm{wt} \% \mathrm{Cu}$ alloy the liquid contains more so-446 lute which leads to more metastable liquid and only parts of $\mathrm{f}_{47}$ the liquid channels merge and form wet GBs, as demonstrated ${ }_{448}$ in Fig. 6.b). Since for Al-4.0wt $\% \mathrm{Cu}, T_{b}-\Delta T_{b}<T_{e}$, the 449 metastable liquid is thus expected to form eutectic phase and 450 thus interphase boundaries. Therefore, the liquid layer formed ${ }_{451}$ is this case cannot continuously extend to $T_{b}-\Delta T_{b}$ as it will be interrupted by interphase boundaries. In principle, the in- ${ }_{453}$ terphase boundaries have extra energy and can remain wet to $_{454}$ certain temperatures as long as their energy is larger than $2 \gamma_{s l} ._{455}$ However, for the $\mathrm{Al}-\mathrm{Al}_{2} \mathrm{Cu}$ eutectics expected to form in this ${ }_{456}$ system, experimental studies [30] show that low-energy semi- ${ }_{457}$ coherent interphase boundaries form, which is not expected to $_{458}$ stay wet to the lowest temperature $T_{b}-\Delta T_{b}$. It can be inferred that compared to the high-angle GB, interphase boundaries in this case are less likely to promote hot cracking.

Solute segregation at emerging GBs depends strongly on ${ }_{462}$ the configuration of solidifying grains (divergent or convergent ${ }_{463}$ growth), as well as the misorientation angles. For small angle ${ }_{464}$ misorientations, GB segregation and misorientaition energy are $_{465}$ coupled. To isolate the effect of segregation alone, high-angle ${ }_{466}$ GBs, with energy independent of misorientation were consid- ${ }_{467}$ ered in PF simulations. The results are shown in Figs. 7 and 8 It was found that divergent growth always leads to higher solute ${ }_{469}$ segregation at intergranular channels than convergent growth. ${ }_{470}$ This difference can be explained by the lateral diffusion in liq- ${ }_{471}$ uid ahead of dendrite arrays. For convergent dendrites which $_{472}$ grow towards each other, there is a strong solute interaction and $_{473}$ a higher solute pile-up in front of tips, which leads to diffusion ${ }_{474}$ in the lateral direction away from the space between the grains. In contrast, divergent primary dendrite stems grow away from ${ }_{476}$ each other, leaving the area between them less saturated with ${ }_{477}$ impurities. This creates a mass sink for solute to diffuse into ${ }_{478}$ the space between the dendrites. also found to be modulated by misorientation angle (Figs. 7 and 8). The solute field in front of two divergent/convergent dendrites can also depend on orientation-dependent primary spacing and side-branch growth for divergent cases. This make the dependence on misorientation complicated. It is plausible, for example, that more side-branches developed with the increasing divergent angle prevent solutes go to further into the space between dendrites and thus GBs segregation decrease.

The data discussed above from our PF simulations suggests that convergent growth conditions lead to lower solute segregation at GBs. For the same GB misorientation, liquid at GBs with higher solute have a lower energy, and can remain stable to lower temperatures. It is expected that short liquid channels will form in this case, which leads to a reduced HCS. This prediction suggest a plausible mechanism for avoiding cracking when welding two components with highly different misorientations, which needs to verified experimentally in the future.

The width of liquid channels at GBs is on the same scale as the solid-liquid interface width. As diffuse interfaces are required to perform large scale PF simulations, it is only possible to resolve the liquid layers at GBs qualitatively below this length, since the physical structure of a liquid channel cannot be modelled at the atomic scale [31]. Nevertheless, the PF simulation can still be used to characterize the magnitude of segregation over a wide range of GB sizes and misorientations, as well as to characterize the length and morphology of extended liquid channels at GBs. PF calculations of such properties can then be used to diagnose HCS in many alloy systems. A proposed future direction for further research is the combination of PF and phase field crystal (PFC) modelling to examine the role of atomic-scale structure on GB energy and segregation, and to predict liquid channel length and morphology at GBs [32-34]. The non-equilibrium segregation and channel morphologies during solidification occupy microstructual length scales, which can be simulated using the PF method. PFC can then use information gathered from PF simulations to examine GB interfaces, at the microscopic scale and on diffusive time scales [35, 36]. 


\subsection{Combining hot cracking models with PF Simulations}

This section develops a methodology to combine information500 from phase field simulations with the the RGD and Kou models501 to identify the composition with the highest HCS in directional502 solidification. The occurrence of hot cracking is caused by the ${ }_{503}$ combined effect of tensile stress and solidification shrinkage.504 Considering columnar growth in one direction with tensile de-505 formation perpendicular to growth direction and liquid feed-506 ing opposite to the growth direction, the RGD model predicts $a_{507}$ pressure drop along a liquid channel given by

$$
\begin{aligned}
\Delta p_{\max } & =\Delta p_{\epsilon}+\Delta p_{s h} \\
& =\frac{180}{\lambda_{i}^{2}} \frac{(1+\beta) \mu}{G} \int_{T_{S}}^{T_{L}} \frac{E f_{s}^{2}}{\left(1-f_{s}\right)^{3}} d T \\
& +\frac{180}{\lambda_{i}^{2}} \frac{v_{T} \beta \mu}{G} \int_{T_{S}}^{T_{L}} \frac{f_{s}^{2}}{\left(1-f_{s}\right)^{2}} d T
\end{aligned}
$$

where $T_{L}, T_{S}$ are the liquidus temperatures at the tips of the dendrtie array and the temperature at the roots of the liquid ${ }^{515}$ channels, respectively. $E(T)$ is given by

$$
E(T)=\frac{1}{G} \int f_{s}(T) \dot{\epsilon}_{p}(T) d T
$$

where $\mu$ is the liquid viscosity, $\beta$ the shrinkage factor, $G$ the 520 thermal gradient, and $v_{T}$ velocity of isotherms. $\lambda_{i}$ is the mi-521 crostructural length scale, with $i=1$ corresponding to the pri-522 mary spacing for cellular growth and $i=2$ to secondary spac-523 ing for dendritic growth. $\dot{\epsilon}_{p}$ is the deformation rate of the solid ${ }^{524}$ perpendicular to the growth direction. Based on the similar ${ }^{25}$ physics as the RGD model, Kou proposed, instead of pressure ${ }^{526}$ drop, using the maximum of the simple index $\left|d T / d \sqrt{f_{s}}\right|\left[37^{527}\right.$ to characterize HCS. We examine both models here.

As analytical models, the RGD and Kou models have tw0529 limitations. The first is that these models are sensitive to the 530 $f_{s}(T)$ information used. As shown in [23], with the increase of 531 back-diffusion and thus variation in $f_{s}(T)$, the HCS peak shifts532 to higher solute contents. The back-diffusion coefficient, how-533 ever, is not easy to determine as its value depends on cooling ${ }_{534}$ rate, microstructure scale and solid diffusion coefficient. More-535 over, mean field type models such as those in Eq. (19) do not536 account for channel length and width variability. In this work we use $f_{s}(T)$ curves calculated from PF simulations, which implicitly incorporates the dependence of back diffusion and microstructure variability statistics along each isotherm.

A second limitation is that the solidification temperature at the roots of liquid channels, $T_{S}$, is not well-defined and its selection can change the $\Lambda$ curve. This temperature enters the RGD model as the lower limit of the integral in Eq. (19), and in the Kou model $\left|d T / d \sqrt{f_{s}}\right|$ reaches its maximum at $T_{S}$. In the original RGD paper, $T_{S}$ is defined as $T\left(f_{s}=0.98\right)$ if eutectic fraction is less than $2 \%$, or $T_{e}$ otherwise. In this case, the maximum HCS is predicted to be $1.4 \mathrm{wt} \% \mathrm{Cu}$. A similar result of $1.5 \mathrm{wt} \% \mathrm{Cu}$ is predicted by Kou with $T_{S}$ defined by where $\sqrt{f_{s}}=0.99$ [37]. Those predictions are close to $1.0 \mathrm{wt} \% \mathrm{Cu}$ observed in casting experiments [38, 39]. However, Most welding experiments observed the peak around $3.0 \mathrm{wt} \% \mathrm{Cu}[40-42]$.

To determine $T_{S}$ from our PF simulation data, we proceed as follows. Referring to Fig. (3), we first determine the liquid fraction at a specific vertical height ( $T$-isotherm) by rastering horizontally, and integrating the total area of liquid $(\phi=-1)$. We denote that liquid fraction by $f_{l}^{\text {channel }}$. The solid fraction at that temperature is then given by $f_{s}^{\text {channel }}=1-f_{l}^{\text {channel }}$. It is noted that in this part of the calculation, liquid droplets and metastable liquid are taken as solid. $f_{s}^{\text {channel }}\left(T_{S}\right) \rightarrow 1$ determines the temperature $T_{S}$. If $f_{s}^{\text {channel }} \rightarrow 1$ below $T_{e}$, then $T_{S}$ is set to $T_{e}$. Once $T_{S}$ is thus established based on the above assumptions, we evaluate Eq. [19], using the actual $f_{s}=1-f_{l}$ into the integrand, where $f_{l}$ is calculated by integrating the total liquid fraction at each isotherm.

Fig. 9 plots the pressure drop calculated Eq. (19), for the $\mathrm{Al}-2.0,3.0$, and $4.0 \mathrm{wt} \% \mathrm{Cu}$ single crystal columnar alloys obtained from PF simulations in this work. The parameters used in Eq. 19 were, $\beta=0.06, \mu=1 \times 10^{-3} \mathrm{~Pa} \mathrm{~s}, \dot{\epsilon_{p}}=10^{-4} \mathrm{~s}^{-1}$. As there is a variabiity in the spacing $\lambda_{1}$, the average values calculated from simulated microstructures are used. There are $\lambda_{1}=3.09,4.00$, and $4.15 \mu \mathrm{m}$ in $\mathrm{Al}-2.0,3.0$, and $4.0 \mathrm{wt} \% \mathrm{Cu}$ alloys, respectively. Fig. 10 presents Kou's HCS parameter $\left(\left|d T / d \sqrt{f_{s}}\right|\right)$ calculated from the same PF simulations used in 
Fig.9. It is found that the $\mathrm{Al}-3.0 \mathrm{wt} \% \mathrm{Cu}$ alloy yields the highest575 HCS index (maximum in the $\Lambda$-curve shown in both Figures).576 For $\mathrm{Al}-2.0 \mathrm{wt} \% \mathrm{Cu}$, the existence of short liquid channels ending 577 at relatively high temperatures is the major reason for low HCS.578 In contrast, a reduced cracking tendency $\mathrm{Al}-4.0 \mathrm{wt} \% \mathrm{Cu}$ can be 579 expected due to a relatively high liquid fraction at $T_{e}$.

Our HCS predictions obtained by combining information ${ }^{581}$ from PF simulations with the RGD/Kou models can be com-582 pared with available experiments. The results shown here used ${ }^{583}$ typical solidification conditions for low speed laser/electron ${ }^{584}$ beam welding processes, for which the maximum HCS is pre-585 dicted to be around 3.0wt $\%$ in $\mathrm{Al}-\mathrm{Cu}$ alloys. These results are ${ }^{586}$ consistent with the work of Michaud et al., who also observed ${ }^{587}$ the peak of $\mathrm{HCS}$ at about $3.5 \mathrm{wt} \% \mathrm{Cu}$ in laser welding of $\mathrm{Al}-\mathrm{Cu}^{588}$ alloys [40]. Other experimental research on hot cracking ten-589 dency of $\mathrm{Al}-\mathrm{Cu}$ alloys in different solidification processes are ${ }^{590}$ summarized in Ref. [3]. In general, most experiments find the ${ }^{591}$ characteristic $\Lambda$-shape curve, as in Figs. 9 and 10 . However, ${ }^{592}$ the nominal composition yielding the highest HCS have also $0^{593}$ been observed to be different in some cases. The reason for this ${ }^{594}$ is likely that grain structures and microstructures within grains ${ }^{595}$ contain non-equilibrium features that can vary from sample to $0^{596}$ sample, and can yield a significant variation in HCS. These fea-597 tures, in turn, depend on solidification processing conditions. ${ }^{598}$ Ideally, a larger ensemble of grain structures would be need to ${ }^{599}$ be simulated to produce a statistically significant measure of ${ }^{600}$ HCS for different nominal compositions.

We can infer that coarse columnar grains are more prone to ${ }^{602}$ hot cracking compared to finer equiaxed grains. Thus, grain re- ${ }^{603}$ finement is an efficient mean to avoid hot cracking [38]. How- ${ }^{604}$ ever, given the variability in model predictions that can arise ${ }^{605}$ due to kinetics and microstructure variability, care needs to be taken to ensure that solidification conditions used to predict the parameters of HCS models adequately capture the macrostructural conditions in experiments. The results of this study sug-608 gest that the combination of quantitative PF simulation data6os with existing hot cracking models can provide a good tool for 610 better predictions of HCS in alloys.
In this work, as we focused on solidification under welding conditions, where microstructures are mainly cellular. Under traditional casting conditions, dendrites are the dominant structure of grains. In terms of effects on HCS, dendrites can be more complicated than cell structure due to side branches. The stochastic feature of sidebranching [43] and the growth and coarsening of secondary arms [44] make coalescence of dendrite structure complex. Moreover, the side branches can nevertheless affect interdendritic flow. This can be seen from the Carman-Kozeny equation in which the permeability of the mushy zone is related to $f_{s}(T)$ and the characteristic length $\lambda_{i}$ by $K=\left[\lambda_{i}^{2}\left(1-f_{s}\right)^{3}\right] /\left[180 f_{s}^{2}\right]$. Normally, the $\lambda_{1}$ is the primary spacing for cell structures and the secondary spacing for dendrites. It is also possible to quantify the interdendritic flow by coupling PF-simulated microstructures with the fluid flow calculations [45].

While the basic mechanisms of hot cracking in 3D are also present in $2 \mathrm{D}$, microstructure connectivity and liquid feeding is more complex in 3D, which can affect HCS. As shown by Tourret et al. [46], there is a difference in the solute diffusion field and final redistribution profiles for 2D versus 3D which can further alter microstructure length. In addition, connectivity of liquid channels can be different in 3D compared to $2 \mathrm{D}$ case [47]. It is plausible that the symmetry of the single and bicrystal grains studied in our work makes it reasonable to compare corresponding 2D and 3D single/bi-crystal systems. This is also consistent with the good comparison of our results to experiments. Due to recent development of computer technology, large-scale 3D phase-field simulations are now viable [46, 48[50]. We are preparing a future 3D work on more complex morphologies using a new 3D parallel, adaptive mesh refinement algorithm to access large scale polycrystalline systems.

\section{Conclusions}

Segregation and morphology of liquid channels during columnar growth of Al-Cu single crystals and bi-crystals were studied using PF simulations. The results show that the backdiffusion is weak in Al-Cu alloys but it can be important for 
other alloys with lower eutectic temperatures. The relative 649 value of the coalescence temperature to the eutectic temper-650 ature were examined in the context of liquid channel formation versus liquid drop coalescence in single crystal columnaro51 growth. Liquid channels are shown to coalesce into droplets ${ }_{652}$ above $T_{e}$ for $\mathrm{Al}-2.0,3.0 \mathrm{wt} \% \mathrm{Cu}$ alloys, while for $\mathrm{Al}-4.0 \mathrm{wt} \% \mathrm{Cu}^{653}$ alloys liquid at channel roots becomes metastable below $T_{e}$ and $^{654}$ can thus transform to eutectic before coalescence. Simulations of high-angle bi-crystal growth under convergent and divergent ${ }_{657}$ growth conditions were examined. It was shown that solute ${ }^{658}$ pile up in front of convergent dendritic crystal leads to lateral ${ }^{600}$ diffusion away from inter-grain channels, which results in less ${ }_{661}$ segregation in the intergranular liquid channels, and ultimately,662 in grain boundaries. For larger misorientation, a shorter inter ${ }^{663}$ granular liquid channel is expected due to lower segregation ${ }^{664}$ which leads to reduced HCS.

This work combined hot cracking models in the literature667 with information about $f_{s}(T)$ computed from a quantitative ${ }^{668}$ PF model of directional solidification. We demonstrated how ${ }^{669}$ this composite numerical modelling combination can be used ${ }_{671}$ to predict the nominal composition giving the highest HCS in ${ }^{672}$ welding of Al-Cu alloys, with result that were consistent with $^{673}$ $\mathrm{Al}-\mathrm{Cu}$ welding experiments. We propose that combining mean- ${ }^{674}$ field type HSC models with input parameters computed from ${ }_{676}$ pre-calculated solidification data sets obtained from PF simula-677 tions, can provide a practical platform for predicting HCS 678 A natural extension of this work is the further development ${ }_{680}$ of a phase-field model that combines the quantitative features of ${ }_{681}$ models such as the one presented here with the self-consistent ${ }^{682}$ nucleation and growth of primary and secondary phases inside ${ }^{683}$ liquid channels. This will be the focus of an upcoming study.

\section{Acknowledgement}

Nikolas Provatas acknowledges financial support by the 689 Canada Research Chairs (CRC) Program. Nan Wang acknowl-690 edges financial support by the National Science Foundation of ${ }^{691}$ China under Grant No.51671160. Lei Wang was supported by $y_{693}$ the China Scholarship Council as a Graduate Trainee at McGillog4
University. We acknowledge Compute Canada and the McGill HPC Centre for computational resources.

\section{References}

[1] W. Kurz, D. Fisher, Fundamentals of solidification, 4th Edition, Trans Tech Publications Ltd, Switzerland, 1998.

[2] J. A. Dantzig, M. Rappaz, Solidification, EPFL press, 2009.

[3] D. Eskin, Suyitno, L. Katgerman, Mechanical properties in the semi-solid state and hot tearing of aluminium alloys, Prog. Mater Sci. 49 (5) (2004) $629-711$.

[4] M. Rappaz, A. Jacot, W. Boettinger, Last-stage solidification of alloys: theoretical model of dendrite-arm and grain coalescence, Metall. Mater. Trans. A 34 (3) (2003) 467-479.

[5] J. Luo, V. Gupta, D. Yoon, H. Meyer III, Segregation-induced grain boundary premelting in nickel-doped tungsten, Appl. Phys. Lett. 87 (23) (2005) 231902.

[6] J. Luo, H. Cheng, K. M. Asl, C. J. Kiely, M. P. Harmer, The role of a bilayer interfacial phase on liquid metal embrittlement, Science 333 (6050) (2011) 1730-1733.

[7] W. J. Boettinger, J. A. Warren, C. Beckermann, A. Karma, Phase-field simulation of solidification, Annu. Rev. Mater. Res. 32 (1) (2002) 163.

[8] L.-Q. Chen, Phase-field models for microstructure evolution, Annu. Rev. Mater. Res. 32 (1) (2002) 113-140.

[9] A. Badillo, C. Beckermann, Phase-field simulation of the columnar-toequiaxed transition in alloy solidification, Acta Mater. 54 (8) (2006) 2015-2026

[10] H. Xing, X. Dong, C. Chen, J. Wang, L. Du, K. Jin, Phase-field simulation of tilted growth of dendritic arrays during directional solidification, Int. J. Heat Mass Transfer 90 (2015) 911-921.

[11] B. Böttger, M. Apel, B. Santillana, D. Eskin, Phase-field modelling of microstructure formation during the solidification of continuously cast low carbon and hsla steels, in: IOP Conference Series: Materials Science and Engineering, Vol. 33, IOP Publishing, 2012, p. 012107.

[12] B. Böttger, G. Schmitz, B. Santillana, Multi-phase-field modeling of solidification in technical steel grades, Trans. Indian Inst. Met. 65 (6) (2012) $613-615$.

[13] B. Böttger, M. Apel, B. Santillana, D. Eskin, Relationship between solidification microstructure and hot cracking susceptibility for continuous casting of low-carbon and high-strength low-alloyed steels: a phase-field study, Metall. Mater. Trans. A 44 (8) (2013) 3765-3777.

[14] N. Ofori-Opoku, N. Provatas, A quantitative multi-phase field model of polycrystalline alloy solidification, Acta Mater. 58 (6) (2010) 2155-2164.

[15] B. Echebarria, R. Folch, A. Karma, M. Plapp, Quantitative phase-field model of alloy solidification, Phys. Rev. E 70 (6) (2004) 061604.

[16] A. Farzadi, M. Do-Quang, S. Serajzadeh, A. Kokabi, G. Amberg, Phasefield simulation of weld solidification microstructure in an $\mathrm{Al}-\mathrm{Cu}$ alloy, Modell. Simul. Mater. Sci. Eng. 16 (6) (2008) 065005. 
[17] W. Read, W. Shockley, Dislocation models of crystal grain boundaries, 743 Phys. Rev. 78 (3) (1950) 275.

[18] P. R. Cantwell, M. Tang, S. J. Dillon, J. Luo, G. S. Rohrer, M. P. Harmer,745 Grain boundary complexions, Acta Mater. 62 (2014) 1-48.

[19] B. Echebarria, A. Karma, S. Gurevich, Onset of sidebranching in direc-747 tional solidification, Phys. Rev. E 81 (2) (2010) 021608.

[20] S.-Z. Lu, J. Hunt, P. Gilgien, W. Kurz, Cellular and dendritic growth749 in rapidly solidified Al-Fe and Al-Cu alloys, Acta Metall. Mater. 42 (5)750 (1994) 1653-1660.

[21] N. Provatas, N. Goldenfeld, J. Dantzig, Adaptive mesh refinement com-752 putation of solidification microstructures using dynamic data structures, 753 J. Comput. Phys. 148 (1) (1999) 265-290.

[22] N. Provatas, N. Goldenfeld, J. Dantzig, Efficient computation of dendritic755 microstructures using adaptive mesh refinement, Phys. Rev. Lett. 80 (15)756 (1998) 3308.

[23] J. Liu, S. Kou, Effect of diffusion on susceptibility to cracking during758 solidification, Acta Mater. 100 (2015) 359-368.

[24] J. Liu, S. Kou, Crack susceptibility of binary aluminum alloys during760 solidification, Acta Mater. 110 (2016) 84-94.

[25] S. Kou, Welding Metallurgy, 2nd Edition, John Wiley \& Sons, Inc., 2003.762

[26] J. Liu, H. P. Duarte, S. Kou, Evidence of back diffusion reducing cracking763 during solidification, Acta Mater. 122 (2017) 47-59.

[27] Suyitno, V. I. Savran, L. Katgerman, D. G. Eskin, Effects of alloy com-765 position and casting speed on structure formation and hot tearing dur-766 ing direct-chill casting of Al-Cu alloys, Metall. Mater. Trans. A 35 (11)767 (2004) 3551

[28] Suyitno, D. Eskin, L. Katgerman, Structure observations related to hot769 tearing of Al-Cu billets produced by direct-chill casting, Mater. Sci. Eng.,770 A 420 (1) (2006) 1-7.

[29] N. Wang, S. Mokadem, M. Rappaz, W. Kurz, Solidification cracking of 772 superalloy single-and bi-crystals, Acta Mater. 52 (11) (2004) 3173-3182.773

[30] B. Cantor, G. Chadwick, The growth crystallography of unidirection-774 ally solidified Al-Al3Ni and Al-A12Cu eutectics, J. Cryst. Growth 23 (1)775 (1974) 12-20.

[31] Y. Mishin, W. Boettinger, J. Warren, G. McFadden, Thermodynamics of 777 grain boundary premelting in alloys. I. phase-field modeling, Acta Mater.778 57 (13) (2009) 3771-3785.

[32] K. Elder, M. Grant, Modeling elastic and plastic deformations in nonequi-780 librium processing using phase field crystals, Phys. Rev. E 70 (5) (2004)781 051605 .

[33] K. Elder, M. Katakowski, M. Haataja, M. Grant, Modeling elasticity in783 crystal growth, Phys. Rev. Lett. 88 (24) (2002) 245701.

[34] N. Provatas, K. Elder, Phase-field methods in materials science and engi-785 neering, John Wiley \& Sons, 2011.

786

[35] H. Emmerich, H. Löwen, R. Wittkowski, T. Gruhn, G. I. Tóth, G. Tegze,787 L. Gránásy, Phase-field-crystal models for condensed matter dynamics on atomic length and diffusive time scales: an overview, Adv. Phys. 61 (6) (2012) 665-743.
[36] N. Provatas, J. Dantzig, B. Athreya, P. Chan, P. Stefanovic, N. Goldenfeld, K. Elder, Using the phase-field crystal method in the multi-scale modeling of microstructure evolution, JOM 59 (7) (2007) 83-90

[37] S. Kou, A simple index for predicting the susceptibility to solidification cracking, Weld. J. 94 (12) (2015) 374S-388S.

[38] J. Spittle, A. Cushway, Influences of superheat and grain structure on hot-tearing susceptibilities of Al-Cu alloy castings, Met. Technol. 10 (1) (1983) 6-13.

[39] I. Novikov, Hot-shortness of Nonferrous Metals and Alloys, Nauka, 1966.

[40] E. Michaud, H. Kerr, D. Weckman, Temporal pulse shaping and solidification cracking in laser welded Al-Cu alloys, in: H. Smartt, J. Johnson, S. S. A. David (Eds.), Trends in Welding Research, ASM International, Materials Park, OH (United States), 1995, p. 154.

[41] W. Pumphrey, J. Lyons, Cracking during the casting and welding of the more common binary aluminium alloys, J. Inst. Met. 74 (9) (1948) 439.

[42] C. Cross, D. Olson, Hot tearing model to assess aluminum weldability, Aluminum AIloys-Their Physical and Mechanical Properties (1986) 1869-1875

[43] A. Karma, W.-J. Rappel, Phase-field model of dendritic sidebranching with thermal noise, Phys. Rev. E 60 (4) (1999) 3614.

[44] D. Kammer, P. Voorhees, The morphological evolution of dendritic microstructures during coarsening, Acta Mater. 54 (6) (2006) 1549-1558.

[45] C. Puncreobutr, A. Phillion, J. Fife, P. Lee, Coupling in situ synchrotron $\mathrm{X}$-ray tomographic microscopy and numerical simulation to quantify the influence of intermetallic formation on permeability in aluminiumsilicon-copper alloys, Acta Mater. 64 (2014) 316-325.

[46] D. Tourret, Y. Song, A. Clarke, A. Karma, Grain growth competition during thin-sample directional solidification of dendritic microstructures: A phase-field study, Acta Mater. 122 (2017) 220-235

[47] M. Sistaninia, A. Phillion, J.-M. Drezet, M. Rappaz, Three-dimensional granular model of semi-solid metallic alloys undergoing solidification: Fluid flow and localization of feeding, Acta Mater. 60 (9) (2012) 39023911.

[48] T. Takaki, S. Sakane, M. Ohno, Y. Shibuta, T. Shimokawabe, T. Aoki, Large-scale phase-field studies of three-dimensional dendrite competitive growth at the converging grain boundary during directional solidification of a bicrystal binary alloy, ISIJ Int. 56 (8) (2016) 1427-1435.

[49] T. Takaki, S. Sakane, M. Ohno, Y. Shibuta, T. Shimokawabe, T. Aoki, Primary arm array during directional solidification of a single-crystal binary alloy: Large-scale phase-field study, Acta Mater. 118 (2016) 230-243.

[50] Y. Shibuta, M. Ohno, T. Takaki, Solidification in a supercomputer: from crystal nuclei to dendrite assemblages, JOM 67 (8) (2015) 1793-1804.

[51] S. Liu, R. Napolitano, R. Trivedi, Measurement of anisotropy of crystalmelt interfacial energy for a binary Al-Cu alloy, Acta Mater. 49 (20) (2001) $4271-4276$ 
Table 1: Physical properties of Al-Cu alloys used in the PF simulations [1] 51]. ${ }_{816}$

\begin{tabular}{ll}
\hline \hline Melting temperature of Al, $T_{m}$ & $933.47 \mathrm{~K}$ \\
Liquidus slope, $m$ & $-2.6 \mathrm{~K} / \mathrm{wt} \%$ \\
Partition coefficient, $k$ & 0.14 \\
Liquid diffusion coefficient, $D_{l}$ & $3.0 \times 10^{-9} \mathrm{~m}^{2} \mathrm{~s}^{-1}$ \\
Solid diffusion coefficient, $D_{s}$ & $3.0 \times 10^{-13} \mathrm{~m}^{2} \mathrm{~s}^{-1}$ \\
Anisotropy of surface energy, $\epsilon_{4}$ & 0.01 \\
Gibbs-Thompson coefficient, $\Gamma$ & $2.4 \times 10^{-7} \mathrm{Km}$ \\
\hline \hline
\end{tabular}

\section{Tables}

\section{List of Figures}

790

a 1 Schematic diagram illustrating the effect of nominal composition on liquid channels of alloys with (a) low, (b) moderate, and (c) high concentration. . . . . . . . . . . . 15

a 2 Grain boundary energy in the phase field model relative to a characteristic solid-liquid interface energy of the model: (a) $\gamma_{g b}^{\max } / \gamma_{s l}$ versus driving force coefficient, and (b) $\gamma_{g b} / \gamma_{s l}$ versus misorientation angle. . . . . . . . . . . . 15 $15_{836}$

3 Typical liquid channel morphologies during single crystal columnar growth of $\mathrm{Al}-\mathrm{Cu}$ alloys with different nominal compositions. The $T_{m}$ (melting), $T_{l}$ (liquidus), $T_{s}$ (solidus), and $T_{e}$ (eutectic) isotherms are indicated. . . . . . . . 16 $16_{841}$

4 Solid fraction $f_{s}$ vs. temperature $T$ curves calculated from PF simulations and from the Scheil equation for $\mathrm{Al}-2.0,3.0$, and $4.0 \mathrm{wt} \% \mathrm{Cu}$ alloys. . . . . . . . . . . . . . . . . 16 $5 \quad f_{s}$ vs. $T$ curves for an Al-3.0wt\% Cu alloy calculated from the PF simulations with different solid diffusion coefficients.

6 Liquid channels in bi-crystal columnar growth of a simulated (a) $\mathrm{Al}-2.0 \mathrm{wt} \% \mathrm{Cu}$ and (b) $\mathrm{Al}-$ $4.0 \mathrm{wt} \% \mathrm{Cu}$ alloy. The shadowed areas are enlarged below to show details. $T_{m}, T_{l}, T_{s}$ and $T_{e}$ have the same meaning as in Figure 3 . . . .
817

818

819

$$
820
$$
6

7 Solute distribution within liquid channels in bi-crystal columnar growth of a simulated Al$2.0 \mathrm{wt} \% \mathrm{Cu}$ alloy for three misorientation an\begin{tabular}{|l|l}
\hline gles, (a) convergent growth (b) divergent growth. 17 & 17
\end{tabular}

a 8 Average solute composition at grain boundaries versus misorientation for the convergent and divergent columnar bi-crystal growth cases of the \begin{tabular}{|lll}
\hline $\mathrm{Al}-2.0 \mathrm{wt} \% \mathrm{Cu}$ alloy data in Figure 77$]$ & $\ldots$ & 18
\end{tabular}

$9 \quad$ Computed solid fraction $\left(f_{s}^{\text {channel }}\right)$ and the corr. responding calculated pressure drop $(\Delta P)$ versus temperature for three nominal alloy compositions. The plots were computed from the \begin{tabular}{|l|l|l|}
\hline single crystal simulation data in Figure 3 & The \\
\hline
\end{tabular} rightmost curves for each alloy correspond to the $\Delta P$, with the maximum of $\Delta P$ illustrated for the case of the $4.0 \mathrm{wt} \% \mathrm{Cu}$ alloy. Dotted points connecting maxima of $\Delta P$ indicate the

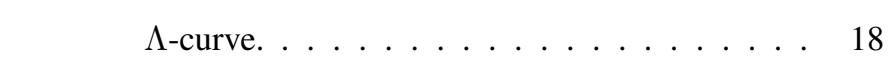
10 Computed solid fraction $\left(f_{s}^{\text {channel }}\right)$ and the corresponding $\left|d T / d \sqrt{f_{s}}\right|$ versus temperature for three nominal alloy compositions. The plots were computed from the single crystal simula\begin{tabular}{|l|l|l|}
\hline 4 tion data in Figure 3 & The rightmost curves for
\end{tabular} each alloy correspond to $\left|d T / d \sqrt{f_{s}}\right|$, with the maximum of $\left|d T / d \sqrt{f_{s}}\right|$ illustrated for the case of the $4.0 \mathrm{wt} \% \mathrm{Cu}$ alloy. Dotted points connect\begin{tabular}{|c|}
\hline ing maxima of $\left|d T / d \sqrt{f_{s}}\right|$ indicate the $\Lambda$-curve. \\
\hline
\end{tabular}

\section{Figures}



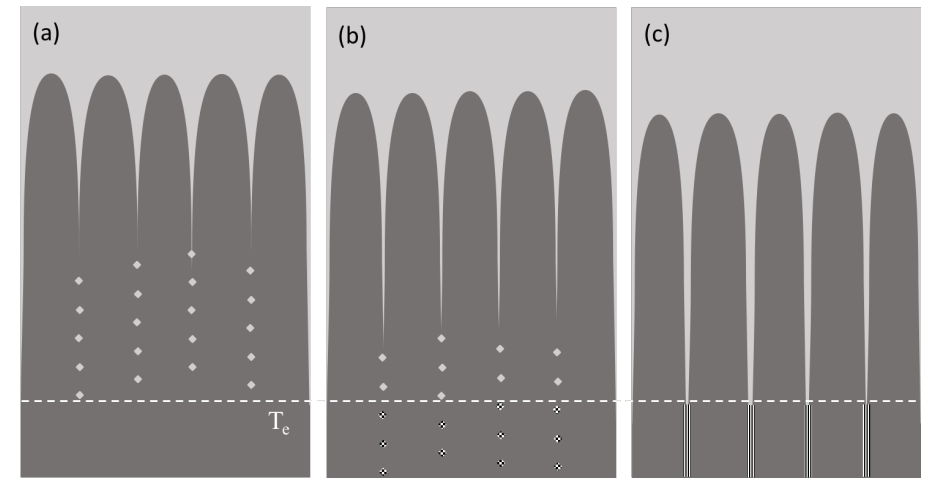

Figure 1: Schematic diagram illustrating the effect of nominal composition on liquid channels of alloys with (a) low, (b) moderate, and (c) high concentration.
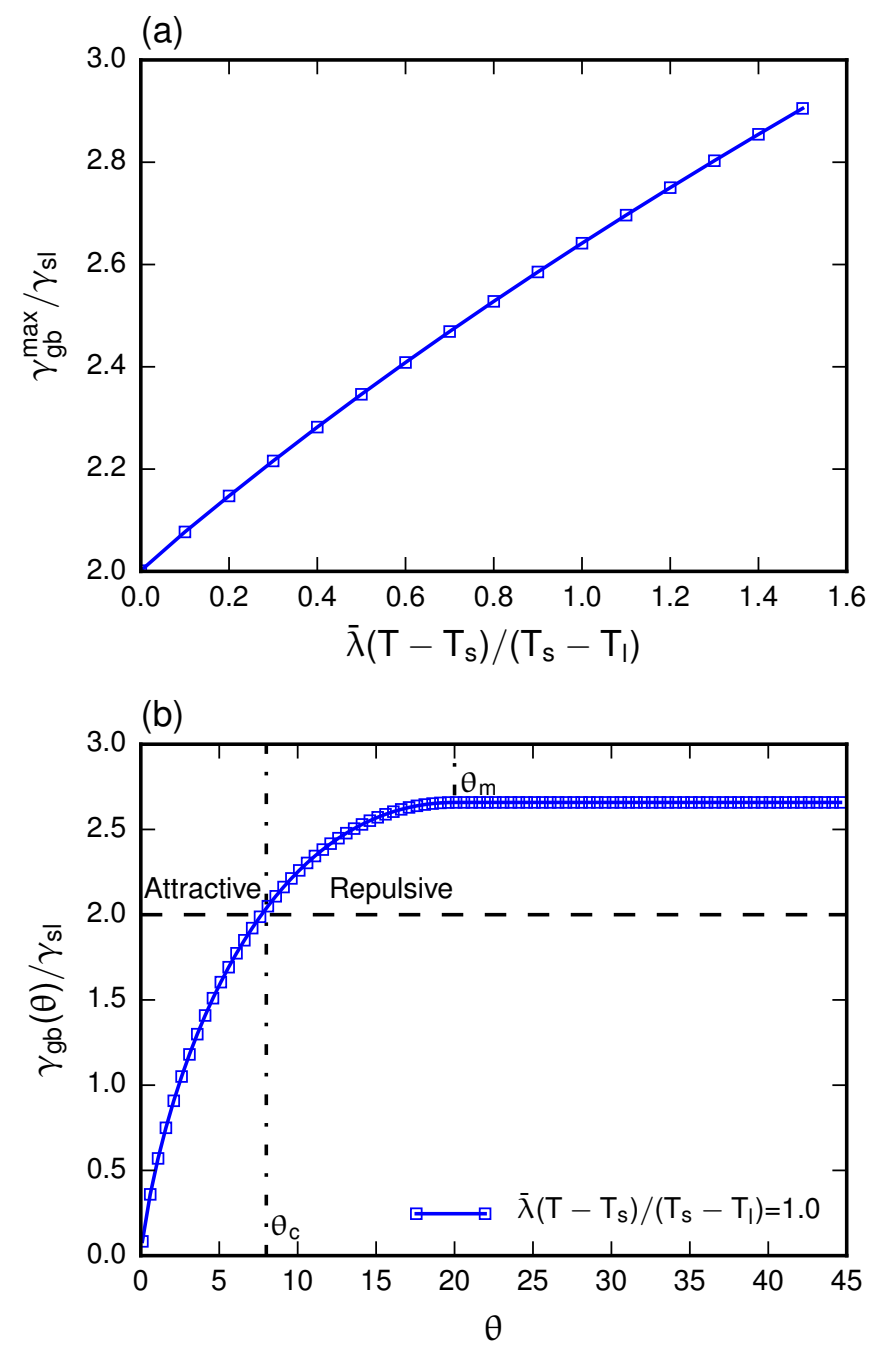

Figure 2: Grain boundary energy in the phase field model relative to a characteristic solid-liquid interface energy of the model: (a) $\gamma_{g b}^{\max } / \gamma_{s l}$ versus driving force coefficient, and (b) $\gamma_{g b} / \gamma_{s l}$ versus misorientation angle. 
(a) Al-2.0wt\%Cu

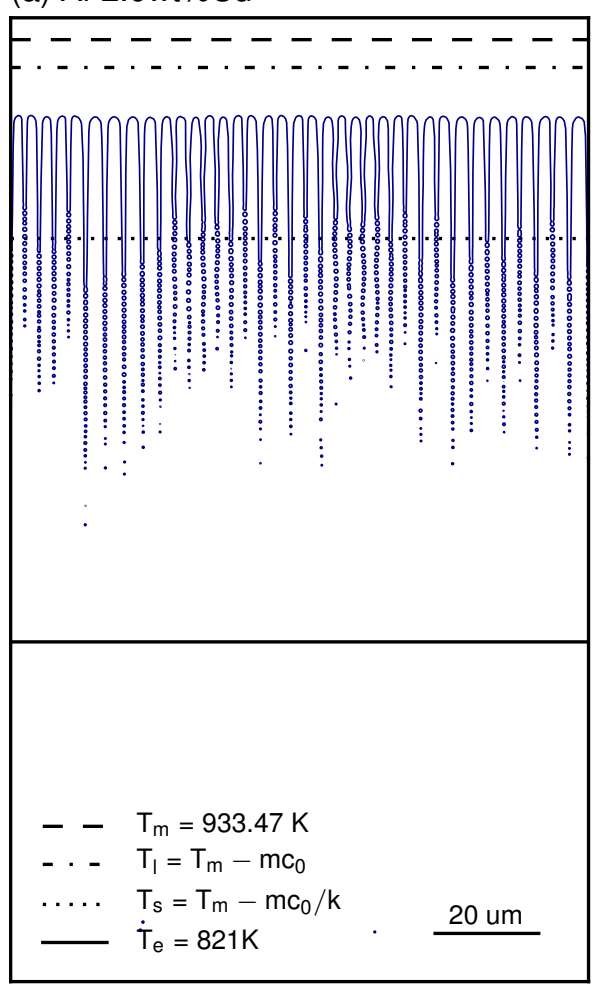

(b) Al-3.0wt\%Cu

(c) $\mathrm{Al}-4.0 \mathrm{wt} \% \mathrm{Cu}$

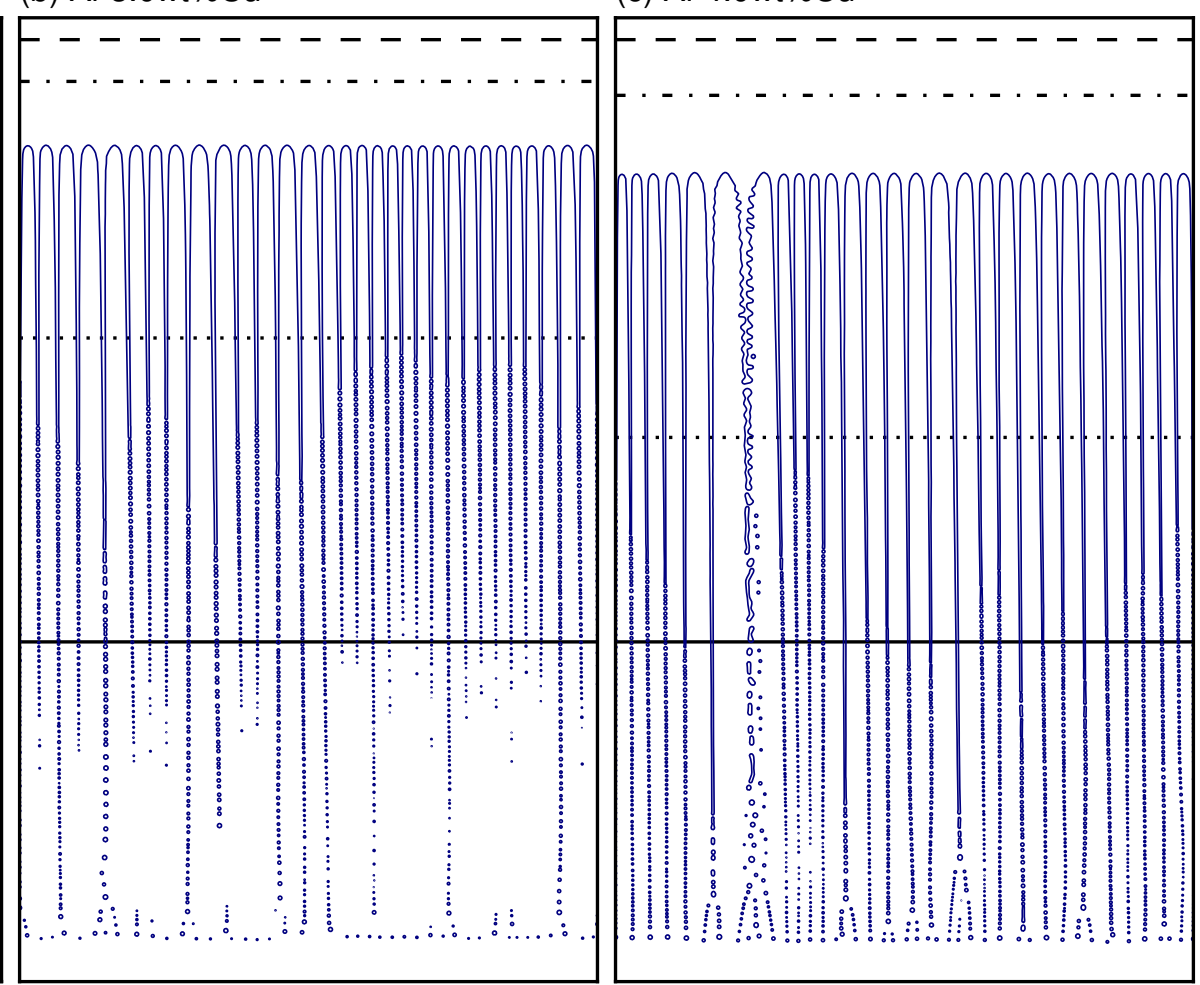

Figure 3: Typical liquid channel morphologies during single crystal columnar growth of Al-Cu alloys with different nominal compositions. The $T_{m}$ (melting), $T_{l}$ (liquidus), $T_{s}$ (solidus), and $T_{e}$ (eutectic) isotherms are indicated.

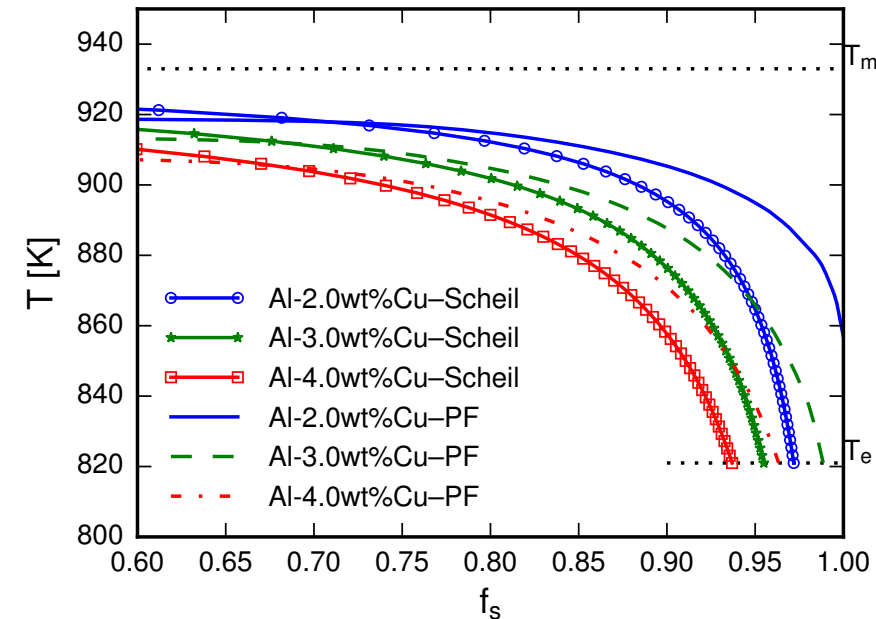

Figure 4: Solid fraction $f_{s}$ vs. temperature $T$ curves calculated from PF simulations and from the Scheil equation for Al-2.0,3.0, and $4.0 \mathrm{wt} \% \mathrm{Cu}$ alloys.

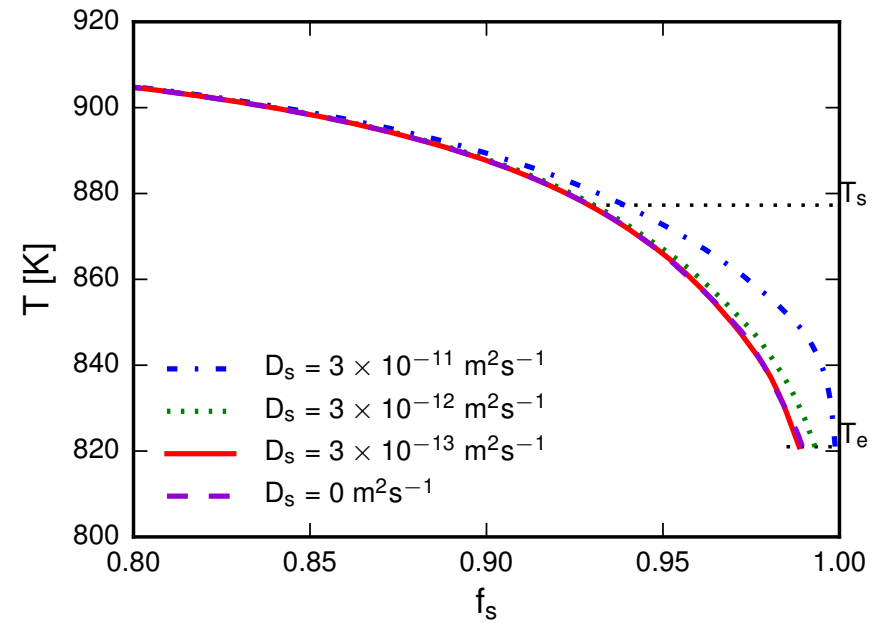

Figure 5: $f_{s}$ vs. $T$ curves for an Al-3.0wt $\% \mathrm{Cu}$ alloy calculated from the $\mathrm{PF}$ simulations with different solid diffusion coefficients. 
(a) Al-2.0wt\%Cu

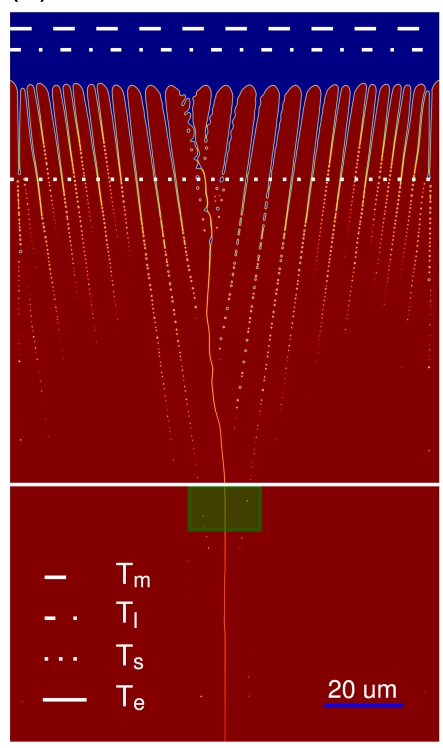

(b) $\mathrm{Al}-4.0 \mathrm{wt} \% \mathrm{Cu}$
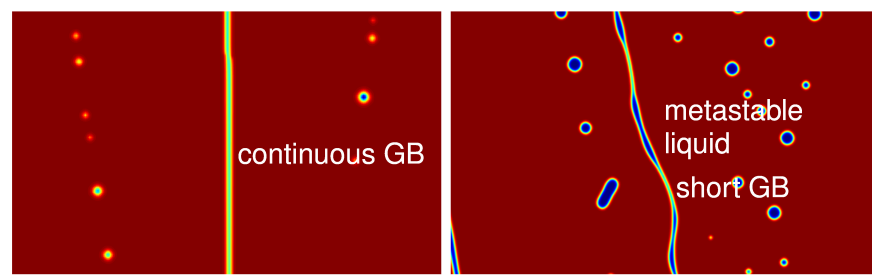

Figure 6: Liquid channels in bi-crystal columnar growth of a simulated (a) Al$2.0 \mathrm{wt} \% \mathrm{Cu}$ and (b) $\mathrm{Al}-4.0 \mathrm{wt} \% \mathrm{Cu}$ alloy. The shadowed areas are enlarged below to show details. $T_{m}, T_{l}, T_{s}$ and $T_{e}$ have the same meaning as in Figure 3 (a) Convergent growth

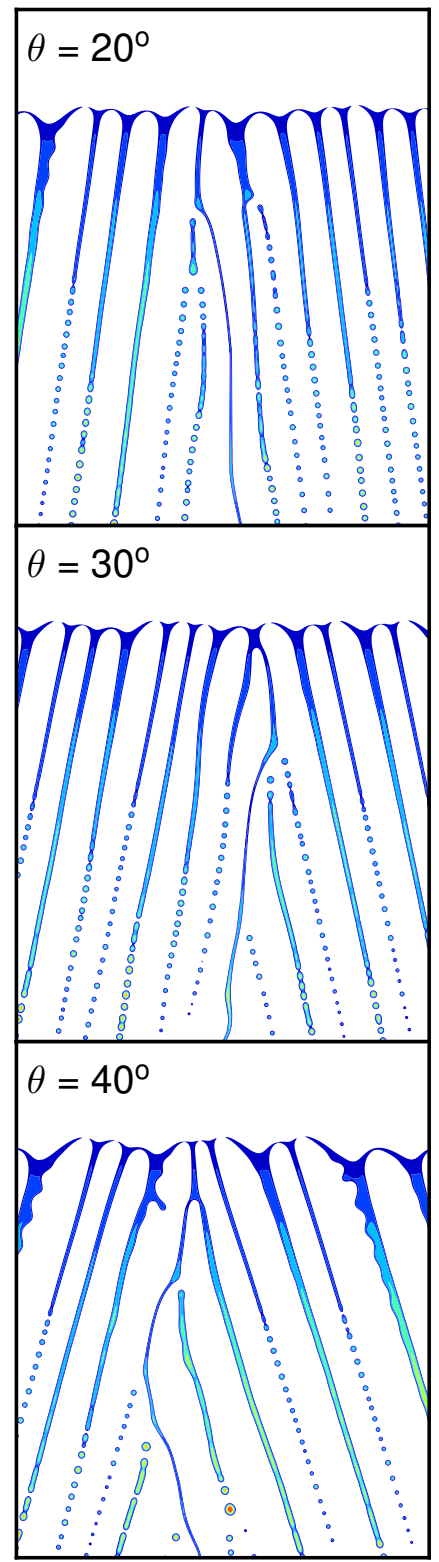

(b) Divergent growth
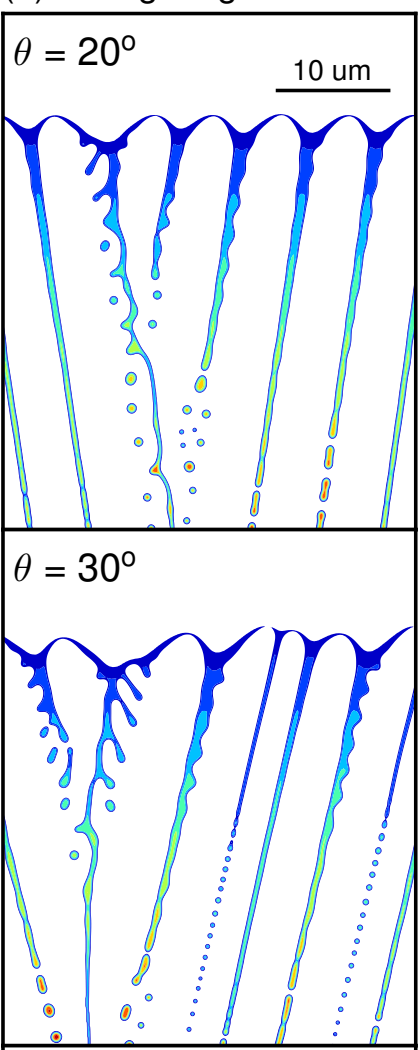

$\theta=40^{\circ}$
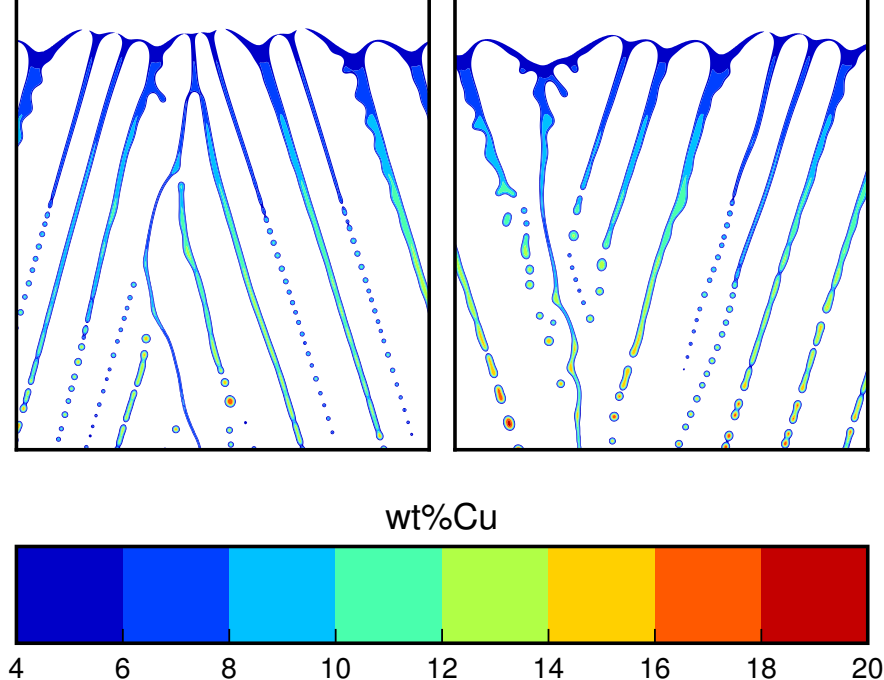

Figure 7: Solute distribution within liquid channels in bi-crystal columnar growth of a simulated $\mathrm{Al}-2.0 \mathrm{wt} \% \mathrm{Cu}$ alloy for three misorientation angles, (a) convergent growth (b) divergent growth. 


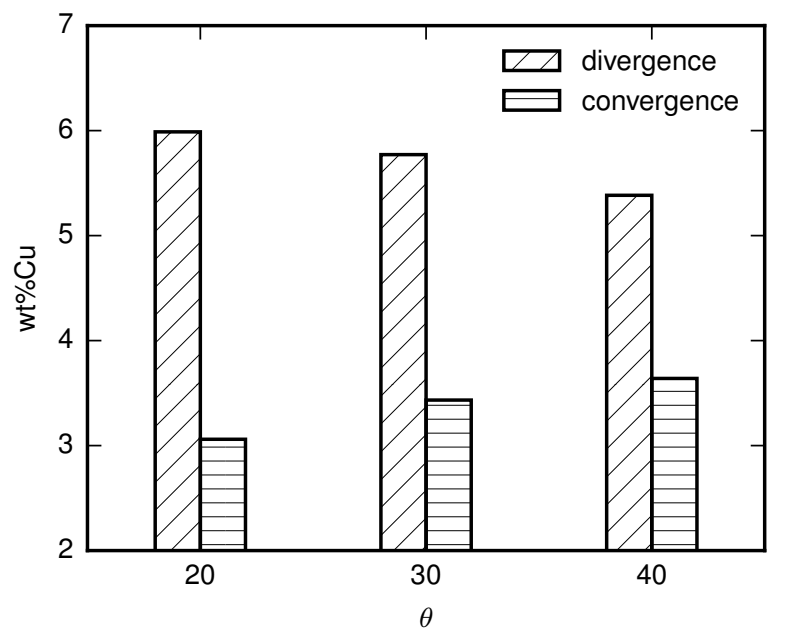

Figure 8: Average solute composition at grain boundaries versus misorientation for the convergent and divergent columnar bi-crystal growth cases of the Al$2.0 \mathrm{wt} \% \mathrm{Cu}$ alloy data in Figure 7

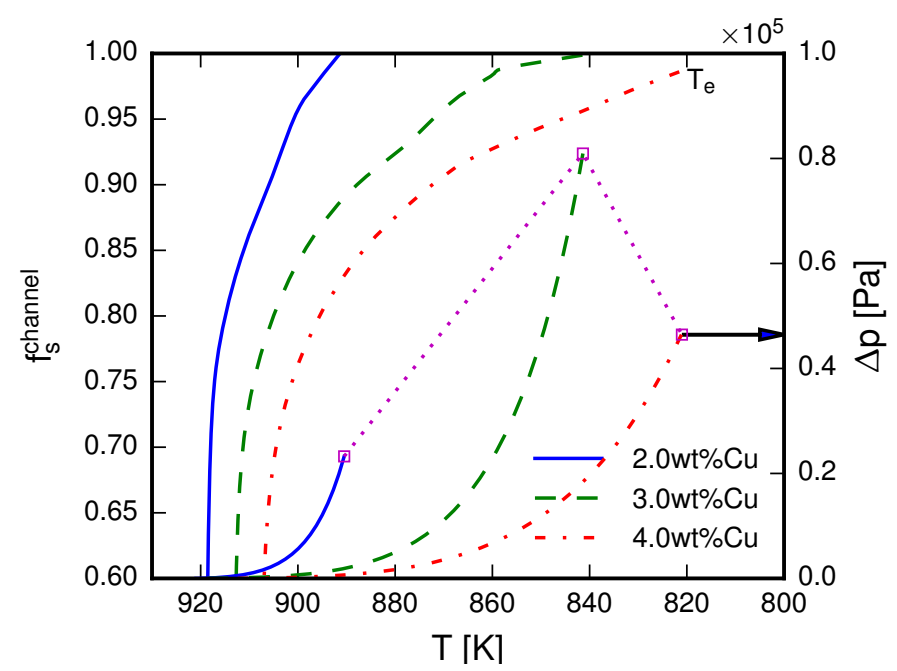

Figure 9: Computed solid fraction $\left(f_{s}^{\text {channel }}\right)$ and the corresponding calculated pressure drop $(\Delta P)$ versus temperature for three nominal alloy compositions. The plots were computed from the single crystal simulation data in Figure 3 The rightmost curves for each alloy correspond to the $\Delta P$, with the maximum of $\Delta P$ illustrated for the case of the $4.0 \mathrm{wt} \% \mathrm{Cu}$ alloy. Dotted points connecting maxima of $\Delta P$ indicate the $\Lambda$-curve.

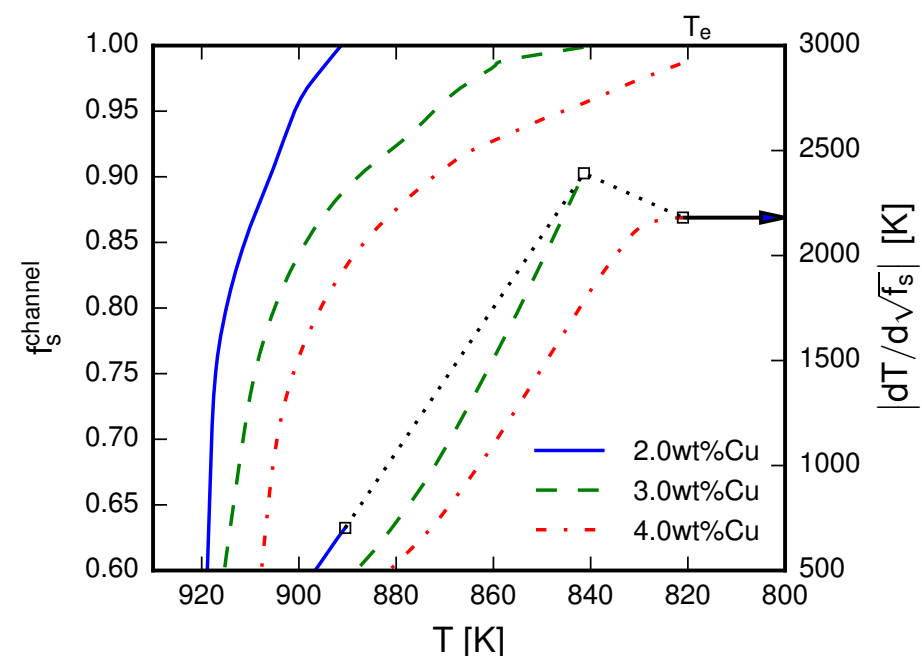

Figure 10: Computed solid fraction $\left(f_{s}^{\text {channel }}\right)$ and the corresponding $\left|d T / d \sqrt{f_{s}}\right|$ versus temperature for three nominal alloy compositions. The plots were computed from the single crystal simulation data in Figure 3 The rightmost curves for each alloy correspond to $\left|d T / d \sqrt{f_{s}}\right|$, with the maximum of $\left|d T / d \sqrt{f_{s}}\right|$ illustrated for the case of the $4.0 \mathrm{wt} \% \mathrm{Cu}$ alloy. Dotted points connecting maxima of $\left|d T / d \sqrt{f_{s}}\right|$ indicate the $\Lambda$-curve. 
